\title{
Female mate recognition and sexual isolation depending on courtship song in Drosophila sechellia and its siblings
}

\author{
Masatoshi Tomaru ${ }^{1 *}$, Hirokazu Yamada ${ }^{2}$ and Yuzuru Oguma ${ }^{2}$ \\ ${ }^{1}$ Drosophila Genetic Resource Center, Kyoto Institute of Technology, Saga-Ippongi-cho, \\ Ukyo-ku, Kyoto 616-8354, Japan \\ ${ }^{2}$ Institute of Biological Sciences, University of Tsukuba, Tsukuba, \\ Ibaraki 305-8572, Japan
}

(Received 22 December 2003, accepted 2 July 2004)

\begin{abstract}
In Drosophila sechellia, females accept males that sing heterospecific songs less than those that do not sing, whereas in $D$. melanogaster and D. simulans, females accept males that sing heterospecific song more than those that do not sing. Here we studied the sexual isolation of $D$. sechellia and its siblings using interspecific hybrids to reveal the mechanisms underlying female mate recognition. The females of hybrids mated more with winged males of the parent species than with wingless ones, suggesting that the discrimination against heterospecific songs by D. sechellia females is recessive. Female preference for courtship songs seems to be inherited additively or semidominantly. In addition, we examined female receptivity without the stimuli of courtship songs by comparing the mating frequencies between the crosses using wingless males and found that it is also inherited additively or semidominantly.
\end{abstract}

Key words: courtship songs, Drosophila, female choice, interspecific hybrids, sexual isolation

\section{INTRODUCTION}

Female mate recognition plays an important role in the sexual isolation of Drosophila. During courtship, both sexes exchange information using several modalities, such as visual, olfactory, and acoustic modalities (Ewing 1983). Courtship in Drosophila is one of the best subjects to study the genetic basis of behavior. However, the genetic basis of female mate recognition responsible for sexual isolation is not yet clarified (e.g., Tan 1946, Zouros 1981, Kyriacou and Hall 1982, Coyne 1996, Noor 1997).

The D. melanogaster complex consists of four closely related species: $D$. melanogaster and $D$. simulans, which are distributed worldwide, and D. sechellia and D. mauritiana, which are found in the islands of Seychelles and Mauritius, respectively (Lachaise et al. 1988). Drosophila sechellia and D. mauritiana diverged allopatrically and they share an ancestor with cosmopolitan $D$. simulans (Lachaise et al. 1988). Drosophila sechellia has a specialized food preference for Morinda citrifolia, although the other species are generalists (Lachaise et al. 1988, R'Kha et al. 1991, Higa and Fuyama 1993). The courtship behavior of the four species of the D. melano-

Edited by Etsuko Matsuura

* Corresponding author. E-mail: tomaru@kit.jp gaster complex has been well described (Spieth 1952, Manning 1959, Cobb et al. 1985, 1986, 1988, 1989). The courtship song emitted by male wing vibration (Shorey 1962) is one of the most significant signals in mating and sexual isolation in the four species of the $D$. melanogaster complex (Bennet-Clark and Ewing 1969, Kyriacou and Hall 1982, Ritchie et al. 1999). The mean interpulse interval of courtship song of $D$. sechellia is $85 \mathrm{~ms}$, which is the longest among the four species of the D. melanogaster complex (Cobb et al. 1989). Drosophila melanogaster, D. simulans and D. mauritiana also have speciesspecific interpulse intervals of 30-35 ms, $50-55 \mathrm{~ms}$ and 35-50 ms, respectively (Ewing and Bennet-Clark 1968, Kyriacou and Hall 1980, Cowling and Burnet 1981, Cobb et al. 1989).

In D. melanogaster, D. simulans and D. mauritiana, females mate with heterospecific males in the songpresent-condition more than in the no-song-condition, whereas the females of $D$. sechellia mate with $D$. melanogaster or $D$. simulans males in the no-song-condition more than in the song-present-condition (Tomaru et al. 2000, Tomaru and Oguma 2000). It seems that female mate recognition depending on courtship songs in $D$. sechellia is different from that in D. melanogaster, D. simulans and D. mauritiana, and the discrimination against heterospecific songs in $D$. sechellia may have appeared only in this lineage during the history of the speciation of 
the $D$. melanogaster complex. Here, we studied the sexual isolation of $D$. sechellia and its siblings using interspecific hybrids to reveal the mechanisms underlying female mate recognition depending on courtship songs. Our primary interest is whether hybrid females reject $D$. melanogaster or D. simulans males that sing. If this is true, the discrimination against heterospecific songs by $D$. sechellia is dominant, as reported in D. ananassae (Doi et al. 2001). If this is not true, we should examine carefully whether or not it is recessive to its sibling species.

\section{MATERIALS AND METHODS}

Drosophila Stocks Drosophila sechellia SS78, D. melanogaster Canton-S and D. simulans Ogasawara were used (Tomaru et al. 2000, Tomaru and Oguma 2000). Each strain was derived from one wild-caught female. Flies were grown on glucose-yeast-cornmeal-agar medium at $24 \pm 1^{\circ} \mathrm{C}$ in a $14: 10 \mathrm{~h}$ light:dark regime (lights on 7:00 -21:00). To produce interspecific hybrid females, virgin $D$. melanogaster females and $D$. sechellia males were crossed (melanogaster-sechellia hybrids). These hybrids were grown at $22 \pm 1^{\circ} \mathrm{C}$ during the larval and pupal stages to prevent temperature-sensitive pupal death, as in the hybrids of D. melanogaster and D. simulans (Sturtevant 1929). Hybrids between $D$. simulans females and D. sechellia males were also produced (simulans-sechellia hybrids); these hybrids were not subjected to a low culture temperature.

Crossability Tests Virgin females and males were collected without anesthesia within $10 \mathrm{~h}$ of eclosion. They were maintained separately in groups of 10 in a vial for 4 to 5 days, at $24 \pm 1^{\circ} \mathrm{C}$, until use. Two days before the experiments, the wings of male flies were cut with microscissors under carbon dioxide anesthesia for 3 min. About half the flies had their wings removed; the rest were used as winged male controls. All the males were subjected to $3 \mathrm{~min}$ of anesthesia, whereas none of the female flies was subjected to anesthesia.

From 9:00 - 14:00, 10 females and 10 wingless or winged males were introduced into a vial ( $30 \mathrm{~mm}$ diameter $\times 105 \mathrm{~mm}$ high) with food. After $0.5,2,5$ or $96 \mathrm{~h}$, we anesthetized the flies and removed the males. The females were dissected within $8 \mathrm{~h}$ and examined for sperm in their spermathecae and ventral receptacle. We repeated each cross five or more times. In some crosses, the differences in mating frequency between wingless males and winged males could be detected in a short duration, but in other crosses a longer duration was required (Tomaru et al. 2000). Thus on the basis of the preliminary crossability tests, we selected an appropriate duration for each crossability test.

If the mating frequencies of the wingless males were identical among the females, we could identify the effects of songs as the differences between the mating frequencies of the winged males. However, when the mating frequencies of the wingless males were different among the crosses, it is better to compare the mating frequencies of winged males considering those of wingless males. On the basis of the mating frequencies of winged and wingless males, we calculated a song effect index (Yamada et al. 2002), that is, (mating frequency of winged males mating frequency of wingless males) / (mating frequency of wingless males). A positive index shows that the females accepted the winged males more than the wingless ones, whereas a negative index shows that the females rejected the winged males more than the wingless ones.

Statistical Tests A chi-square test with Cochran correction (Zar 1984) was applied to detect the differences in the mating frequency between the crosses of winged and wingless males. To examine the mating frequencies across the species and hybrids, we made pairwise comparisons using a chi-square test with Cochran correction or a Fisher exact probability test by the sequential Bonferroni technique (Rice 1989) with $k=3$.

\section{RESULTS}

Effects of courtship songs Females of D. sechellia mated with heterospecific winged males less than with wingless ones (Tables 1-2 and 1-3). In the other crosses, females of pure species and hybrids mated with winged males more than with wingless ones, but this was not significant in the intraspecific crosses of $D$. melanogaster (Table 1-4) and the 2-h crosses of D. simulans females and D. sechellia males (Table 1-7).

Since hybrid females did not reject $D$. melanogaster or D. simulans males that sing courtship songs (Tables 1-10 and 1-12), it seems less likely that the discrimination against heterospecific songs by $D$. sechellia females is dominant. However, we cannot conclude that the trait is recessive without carefully examining the female mate recognition. We first compared the effects of $D$. sechellia courtship songs across the females of pure species. Next we examined the female preference for $D$. sechellia songs across the females of different species and hybrids with the help of song effect indices. Then we examined the receptivity of the females across all the species and hybrids.

Females of pure species and males of $D$. sechellia The indices of the effects of $D$. sechellia songs (Table 1) were the highest in $D$. sechellia females $(\infty)$, the second highest in D. melanogaster females (1.53) and the lowest in $D$. simulans females (0.55). In the crosses with winged $D$. sechellia males (Table 2-1), the mating frequency of $D$. sechellia was the highest, that of $D$. melan- 
Table 1. Effects of courtship song on mating frequency for females of pure species and hybrids

\begin{tabular}{|c|c|c|c|c|c|c|}
\hline Female & Male & $\begin{array}{c}\text { Mating } \\
\text { frequency } \\
(\%)\end{array}$ & $N$ & $\begin{array}{l}\text { Crossing } \\
\text { time }(\mathrm{h})\end{array}$ & $\chi_{\mathrm{c}^{\prime}}^{2}$ & $\begin{array}{c}\text { Song effect } \\
\text { index }\end{array}$ \\
\hline \multirow[t]{2}{*}{ 1. D. sechellia } & Wingless $D$. sechellia & 0.0 & 80 & 2 & $103.918 * * *$ & $\infty$ \\
\hline & Winged $D$. sechellia & 78.8 & 80 & & & \\
\hline \multirow[t]{2}{*}{ 2. D. sechellia } & Wingless $D$. melanogaster & 22.9 & 70 & 96 & $15.065 * * *$ & -0.94 \\
\hline & Winged D. melanogaster & 1.4 & 70 & & & \\
\hline \multirow[t]{2}{*}{ 3. D. sechellia } & Wingless $D$. simulans & 8.3 & 120 & 96 & $10.435 * *$ & -1 \\
\hline & Winged D. simulans & 0.0 & 120 & & & \\
\hline \multirow[t]{2}{*}{ 4. D. melanogaster } & Wingless D. melanogaster & 64.0 & 50 & 0.5 & 2.380 & 0.22 \\
\hline & Winged D. melanogaster & 78.0 & 50 & & & \\
\hline \multirow[t]{2}{*}{ 5. D. melanogaster } & Wingless $D$. sechellia & 25.0 & 80 & 2 & $22.970 * * *$ & 1.53 \\
\hline & Winged D. sechellia & 63.3 & 79 & & & \\
\hline \multirow[t]{2}{*}{ 6. D. simulans } & Wingless $D$. simulans & 53.2 & 79 & 0.5 & $4.513 *$ & 0.31 \\
\hline & Winged D. simulans & 69.6 & 79 & & & \\
\hline \multirow[t]{2}{*}{ 7. D. simulans } & Wingless $D$. sechellia & 14.3 & 140 & 2 & 2.900 & 0.55 \\
\hline & Winged D. sechellia & 22.1 & 140 & & & \\
\hline \multirow[t]{2}{*}{ 8. D. simulans } & Wingless $D$. sechellia & 22.0 & 100 & 5 & $10.051 * *$ & 0.95 \\
\hline & Winged D. sechellia & 43.0 & 100 & & & \\
\hline \multirow[t]{2}{*}{ 9. mel-sec } & Wingless D. sechellia & 4.3 & 69 & 2 & $6.702 * *$ & 3.65 \\
\hline & Winged $D$. sechellia & 20.0 & 70 & & & \\
\hline \multirow[t]{2}{*}{ 10. mel-sec } & Wingless $D$. melanogaster & 1.7 & 60 & 2 & $11.644 * * *$ & 11.76 \\
\hline & Winged D. melanogaster & 21.7 & 60 & & & \\
\hline \multirow[t]{2}{*}{ 11. sim-sec } & Wingless $D$. sechellia & 10.0 & 60 & 2 & $49.811 * * *$ & 6.46 \\
\hline & Winged D. sechellia & 74.6 & 59 & & & \\
\hline \multirow[t]{2}{*}{ 12. sim-sec } & Wingless $D$. simulans & 28.3 & 60 & 2 & $45.245 * * *$ & 2.16 \\
\hline & Winged D. simulans & 89.7 & 58 & & & \\
\hline
\end{tabular}

mel-sec: hybrids from crosses between D. melanogaster females and D. sechellia males. sim-sec: hybrids from crosses between $D$. simulans females and $D$. sechellia males. $\chi_{\mathrm{c}}^{2}$ : chi-square value with Cochran correction.

$* P<0.05$, ** $P<0.01$, *** $P<0.001$.

ogaster the second highest, and that of $D$. simulans the lowest. In the crosses with wingless $D$. sechellia males (Table 2-2), the mating frequencies of the D. melanogaster and $D$. simulans females were significantly higher than that of $D$. sechellia females.

Preference for D. sechellia songs To examine the preference for $D$. sechellia songs, the comparison of the mating frequencies of winged $D$. sechellia males across the females of all the species (Tables 2-3 and 2-5) seems not appropriate, since mating frequencies significantly differed across the females in the crosses using wingless $D$. sechellia males. Therefore using the song effect indices from the 2-h crosses, we compared the effects of $D$. sechellia songs across the females.

In the crosses of the females of $D$. sechellia, D. melanogaster and their hybrids, the indices of the effects of $D$. sechellia songs (Table 1) were the highest in the D. sech- ellia females $(\infty)$, the second highest in the hybrid females (3.65) and the lowest in the D. melanogaster females (1.53), suggesting that $D$. sechellia songs affect $D$. sechellia females more to accept a courting male, D. melanogaster females less and hybrids intermediately. In the crosses of the females of $D$. sechellia, $D$. simulans and their hybrids, the indices of effects of $D$. sechellia song (Table 1) were the highest in D. sechellia females $(\infty)$, the second highest in hybrid females (6.46) and the lowest in $D$. simulans females (0.55), suggesting that $D$. sechellia songs affect more $D$. sechellia females, less $D$. simulans females and intermediately hybrids. Therefore, the preference for $D$. sechellia songs in hybrid females is intermediate between their parent species.

Female receptivity without song stimuli In the crosses using wingless males, it can be assumed that mating frequency is not affected by courtship songs. To 
Table 2. Comparison of mating frequencies across females in crosses with $D$. sechellia males

\begin{tabular}{ccc}
\hline \hline Male & $\begin{array}{c}\text { Female } \\
\text { (Mating frequency }(\%))\end{array}$ & $\chi^{2}{ }^{\prime}$ for pairwise comparisons \\
\hline
\end{tabular}

Crosses with females of pure species

\begin{tabular}{|c|c|c|c|c|c|c|}
\hline & $\sec$ & mel & $\operatorname{sim}$ & sec vs. mel & sec vs. sim & $s e c$ vs. $\operatorname{sim}$ \\
\hline 1. Winged $D$. sechellia & 78.8 & 63.3 & 22.1 & $4.404 *$ & $65.199 *$ & $35.704 *$ \\
\hline 2. Wingless $D$. sechellia & 0.0 & 25.0 & 14.3 & $22.857 *$ & $11.646 *$ & 3.301 \\
\hline
\end{tabular}

Comparison between females of $D$. sechellia, D. melanogaster and their hybrids and males of D. sechellia

\begin{tabular}{|c|c|c|c|c|c|c|}
\hline & sec & mel-sec & mel & sec vs. mel & mel-sec vs. sec & mel-sec vs. mel \\
\hline 3. Winged D. sechellia & 78.8 & 20.0 & 63.3 & $4.404 *$ & $49.562 *$ & $28.149 *$ \\
\hline 4. Wingless $D$. sechellia & 0.0 & 4.3 & 25.0 & $22.857 *$ & 1.687 & $11.631 *$ \\
\hline
\end{tabular}

Comparison between females of D. sechellia, D. simulans and their hybrids and males of D. sechellia

\begin{tabular}{|c|c|c|c|c|c|c|}
\hline & sec & sim-sec & $\operatorname{sim}$ & sec vs. sim & sim-sec vs. sec & sim-sec vs. sim \\
\hline 5. Winged D. sechellia & 78.8 & 74.6 & 22.1 & $65.199 *$ & 0.166 & $47.421 *$ \\
\hline 6. Wingless $D$. sechellia & 0.0 & 10.0 & 14.3 & $11.646 *$ & $\mathrm{P}=0.0053^{\dagger}$ & 0.473 \\
\hline
\end{tabular}

The mating frequencies are from Table 1.

sec: D. sechellia, mel: D. melanogaster, sim: D. simulans.

mel-sec: hybrids from crosses between $D$. melanogaster females and $D$. sechellia males.

sim-sec: hybrids from crosses between $D$. simulans females and $D$. sechellia males.

$\chi_{\mathrm{c}}^{2}$ : chi-square value with Cochran correction.

'Fisher exact probability.

A chi-square value (or Fisher exact probability) with an asterisk is significant at the 0.05 level after sequential

Bonferroni correction $(k=3)$.

examine female receptivity without the song stimuli, we compared mating frequency across the females of the crosses with wingless $D$. sechellia males. In the crosses of the females of $D$. sechellia, D. melanogaster and their hybrid, the mating frequency of the hybrid females was intermediate between those of $D$. sechellia and D. melanogaster females (Table 2-4), but those of the hybrid and $D$. sechellia did not significantly differ. In the crosses of the females of $D$. sechellia, D. simulans and their hybrid, the mating frequency of $D$. sechellia females was the lowest; the hybrid females had an intermediate mating frequency between those of their parent species (Table 2-6), although it was not significantly different from that of $D$. simulans females.

The comparison across the females of the crosses with wingless $D$. melanogaster or $D$. simulans males will provide useful information, although the crossing times were different among the crosses. The wingless $D$. melanogaster males mated more with the D. melanogaster females $(64.0 \%, 0.5$-h cross, Table 1-4) but less with melanogaster-sechellia hybrid (1.7\%, 2-h cross, Table 1-10) and $D$. sechellia females $(22.9 \%, 96$-h cross, Table 12 ). In the crosses with wingless $D$. simulans males, the mating frequency of $D$. sechellia females was the lowest (8.3\%, 96-h cross, Table 1-3), that of the simulans-sechellia hybrid females the second $(28.3 \%, 2$-h cross, Table 112 ) and $D$. simulans females the highest $(53.2 \%, 0.5-\mathrm{h}$ cross, Table 1-6). It seems that the D. melanogaster or
D. simulans females accept the wingless males more than the $D$. sechellia females.

\section{DISCUSSION}

The $D$. sechellia females mated with the winged $D$. sechellia males more than with the wingless ones, whereas they mated with the winged heterospecific $(D$. melanogaster and $D$. simulans) males less than with the wingless ones (Table 1). In contrast, the D. melanogaster and $D$. simulans females mated with the winged males more than with the wingless ones, even if the males were heterospecific $D$. sechellia. The melanogaster-sechellia hybrid and simulans-sechellia hybrid females also mated with the winged males of their parent species more than with the wingless ones. It seems that the discrimination against heterospecific songs in $D$. sechellia is recessive in $D$. melanogaster and $D$. simulans, or it is maternally inherited. This is in contrast to the case in which the discrimination against heterospecific songs in D. ananassae is dominant in D. pallidosa (Doi et al. 2001).

If the rejection of the songs of D. melanogaster and $D$. simulans is observed in hybrids, we can conclude that the discrimination against heterospecific songs in $D$. sechellia is dominant. In such a case, the hybrid female judges that the song is heterospecific and she discriminates against the "heterospecific" song; then she decides to 
reject the courting male. However, if the rejection is not observed in hybrids, as in our present study, we should carefully examine female mate recognition before we can conclude that the discrimination against heterospecific songs in $D$. sechellia is recessive. If the preference for the $D$. sechellia song is recessive to $D$. melanogaster and $D$. simulans song, the hybrid female judges that the song is conspecific, and then she can copulate well with the winged males of their parent species. The preference function of hybrids is intermediate between those of the parent species if it is additively inherited. Drosophila melanogaster and $D$. simulans females mate well with a male whose song parameters are species-specific values (about $34 \mathrm{~ms}$ of interpulse interval and $55 \mathrm{~s}$ of its fluctuation for D. melanogaster and about $55 \mathrm{~ms}$ of interpulse interval and $35 \mathrm{~s}$ of its fluctuation for $D$. simulans) and they prefers less long or short inter-pulse intervals (Bennet-Clark and Ewing 1969, Kyriacou and Hall 1982). In D. melanogaster females, an artificially synthesized courtship song of the $D$. sechellia type enhances mating more than no sound, but less than the $D$. melanogaster type (Ritchie et al. 1999). The indices of $D$. sechellia song effects in the hybrid females were intermediate between those of the parent species (Table 1). In addition, in the crosses of $D$. melanogaster or D. simulans males, the song effect indices in the hybrid females were relatively high (11.76 for melanogaster-sechellia hybrids and 2.16 for simulans-sechellia hybrids, respectively) and comparable to the indices of $D$. sechellia song effects in hybrids (3.65 for melanogaster-sechellia hybrids and 6.46 for simulans-sechellia hybrids, respectively). Therefore, the female preference for songs in the hybrids seems intermediate between those of the parent species.

Another factor affecting female mate recognition is basic receptivity that is not affected by courtship songs. Assuming that the mating frequency of the cross between females and wingless males indicates the receptivity of the females who did not receive courtship song stimuli, the $D$. melanogaster and $D$. simulans females have higher receptivity than the $D$. sechellia females (Table 2-2). The mating frequencies of the crosses between the females of the hybrids and wingless males were intermediate between those of the parent species (Tables 2-4 and 2-6), suggesting that the hybrids have an intermediate receptivity between those of the parent species. It is therefore likely that basic receptivity is inherited additively or semidominantly.

Although it is possible that wingless $D$. sechellia males court heterospecific females less than conspecific females, the mating frequencies of wingless $D$. sechellia males were higher in the cross with heterospecific females than in that with conspecific females (Table 2-2). During the crossability tests, we often observed that $D$. sechellia males actively courted heterospecific females. Thus the D. sechellia males court heterospecific females well. The wing vibration of males produces sound (Shorey 1962), however, it may also act as a visual signal. The sound receptors of Drosophila are aristae, feather-like branched bristles extending from the third segment of the antennae. An arista rotates to transmit sound vibration to Johnston's organ in the second segment (Manning 1967, Burnet et al. 1971, Göpfer and Robert 2001). We showed that the decrease of the mating frequency of the wingless males are due to the absence of sound as determined using females whose aristae were removed (Tomaru et al. 2000). The female responses of the strains used in this study were found to be species-specific using several strains of these species (Tomaru et al. 2000, Tomaru and Oguma 2000). Since the duration of confinement is longer in some interspecific crosses than in conspecific crosses, the present study may involve many uncontrollable factors affecting female mate recognition. By comparing mating frequency between the winged and wingless males for each experiment between the species and the hybrids, we can control the effects that pheromones and other behavioral factors may play in mate recognition.

We previously proposed that the discrimination against heterospecific songs may be brought about by the reinforcement of reproductive isolation in the $D$. auraria complex (Tomaru et al. 1995). Reinforcement and/or reproductive character displacement is one of the possible driving forces shaping the mate preference function, during the sexual isolation of $D$. sechellia was established. One of the other possible scenarios is that the sexual isolation of $D$. sechellia may have been brought about as a by-product of sexual selection, as proposed in the case of D. simulans and D. mauritiana (Cobb et al. 1988).

We thank K. Sawamura for his advice and suggestions. This work was supported partly by a Grant-in-Aid for Young Scientists (B) (No. 15770154) to M. T. from the Ministry of Education, Culture, Sports, Science and Technology of Japan.

\section{REFERENCES}

Bennet-Clark, H. C. and Ewing, A. W. (1969) Pulse interval as a critical parameter in the courtship song of Drosophila melanogaster. Anim. Behav. 17, 755-759.

Burnet, B., Connolly, K. and Dennis, L. (1971) The function and processing of auditory information in the courtship behaviour of Drosophila melanogaster. Anim. Behav. 19, 409415.

Cobb, M., Connolly, K. and Burnet, B. (1985) Courtship behaviour in the melanogaster species sub-group of Drosophila. Behaviour 95, 203-231.

Cobb, M., Burnet, B. and Connolly, K. (1986) The structure of courtship in the Drosophila melanogaster species sub-group. Behaviour 97, 182-212.

Cobb, M., Burnet, B. and Connolly, K. (1988) Sexual isolation and courtship behavior in Drosophila simulans, D. mauritiana, and their interspecific hybrids. Behav. Genet. 18, 211-225. 
Cobb, M., Burnet, B., Blizard, R. and Jallon, J.-M. (1989) Courtship in Drosophila sechellia: its structure, functional aspects, and relationship to those of other members of the Drosophila melanogaster species subgroup. J. Insect Behav. 2, 63-89.

Cowling, D. E. and Burnet, B. (1981) Courtship songs and genetic control of their acoustic characteristics in sibling species of the Drosophila melanogaster subgroup. Anim. Behav. 29, 924-935.

Coyne, J. A. (1996) Genetics of sexual isolation in male hybrids of Drosophila simulans and D. mauritiana. Genet. Res. 68, 211-220.

Doi, M, Matsuda, M., Tomaru, M., Matsubayashi, H. and Oguma, Y. (2001) A locus for female discrimination behavior causing sexual isolation in Drosophila. Proc. Natl. Acad. Sci. USA 98, 6714-6719.

Ewing, A. and Bennet-Clark, H. C. (1968) The courtship songs of Drosophila. Behaviour 31, 288-301.

Ewing, A. (1983) Functional aspects of Drosophila courtship. Biol. Rev. 58, 275-292.

Göpfer, M. C. and Robert, D. (2001) Turning the key on Drosophila audition. Nature 411, 908.

Higa, I. and Fuyama, Y. (1993) Genetics of food preference in Drosophila sechellia. I. Responses to food attractants. Genetica 88, 129-136.

Kyriacou, C. P. and Hall, J. (1980) Circadian rhythm mutations in Drosophila melanogaster affect short-term fluctuations in the male's courtship song. Proc. Natl. Acad. Sci. USA 77, 6729-6733.

Kyriacou, C. P. and Hall, J. (1982) The function of courtship song rhythms in Drosophila. Anim. Behav. 30, 794-801.

Lachaise, D., Cariou, M.-L., David, J. R., Lemeunier, F., Tsacas, L. and Ashburner, M. (1988) Historical biogeography of the Drosophila melanogaster species subgroup. Evol. Biol. 22, 159-225.

Manning, A. (1959) The sexual isolation between Drosophila melanogaster and Drosophila simulans. Anim. Behav. 7, $60-65$.

Manning, A. (1967) Antennæ and sexual receptivity in Drosophila melanogaster females. Science 158, 136-137.

Noor, M. A. F. (1997) Genetics of sexual isolation and courtship dysfunction in male hybrids of Drosophila pseudoobscura and Drosophila persimilis. Evolution 51, 816-832.

Rice, W. R. (1989) Analyzing tables of statistical tests. Evolution 43, 223-225.

Ritchie, M. G., Harlsey, E. J. and Gleason, J. M. (1999) Drosophila song as a species-specific mating signal and the behavioural importance of Kyriacou and Hall cycles in $D$. melanogaster song. Anim. Behav. 58, 649-657.

R'Kha S., Capy, P. and David, J. R. (1991) Host-plant specialization in the Drosophila melanogaster species complex: a physiological, behavioral, and genetical analysis. Proc. Natl. Acad. Sci. USA 88, 1835-1859.

Shorey, H. H. (1962) Nature of the sound produced by Drosophila melanogaster during courtship. Science 137, 677-678.

Spieth, H. T. (1952) Mating behavior within the genus Drosophila (Diptera). Bull. Am. Mus. Nat. Hist. 99, 395-474.

Sturtevant, A. H. (1929) The genetics of Drosophila simulans. Publs. Carnegie Instn. 399, 1-62.

Tan, C. C. (1946) Genetics of sexual isolation between Drosophila pseudoobscura and Drosophila persimilis. Genetics 31, 558-573.

Tomaru, M., Matsubayashi, H. and Oguma, Y. (1995) Heterospecific inter-pulse intervals of courtship song elicit female rejection in Drosophila biauraria. Anim. Behav. 50, 905914.

Tomaru, M., Doi, M., Higuchi, H. and Oguma, Y. (2000) Courtship song recognition in the Drosophila melanogaster complex: heterospecific songs make females receptive in $D$. melanogaster but not in D. sechellia. Evolution 54, 12861294.

Tomaru, M. and Oguma, Y. (2000) Mate choice in Drosophila melanogaster and D. sechellia: criteria and their variation depending on courtship song. Anim. Behav. 60, 797-804.

Yamada, H., Matsuda, M. and Oguma, Y. (2002) Genetics of sexual isolation based on courtship song between two sympatric species: Drosophila ananassae and D. pallidosa. Genetica 116, 225-237.

Zar, J. H. (1984) Biostatistical Analysis 2nd edn. Englewood Cliffs. N.J.: Prentice-Hall.

Zouros, E. (1981) The chromosomal basis of sexual isolation in two sibling species of Drosophila: D. arizonensis and $D$. mojavensis. Genetics 97, 703-718. 